Research Paper

\title{
Hsa-let-7e-5p Inhibits the Proliferation and Metastasis of Head and Neck Squamous Cell Carcinoma Cells by Targeting Chemokine Receptor 7
}

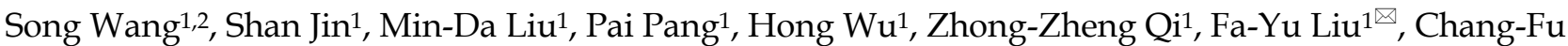 \\ Sun ${ }^{1 凶}$ \\ 1. Department of Oromaxillofacial-Head and Neck Surgery, Oral Maxillofacial Surgery, School of Stomatology, China Medical University, Shenyang, \\ Liaoning, 110002, \\ 2. Department of Stomatology, the 4th Affiliated Hospital of China Medical University, Shenyang, Liaoning, 110034, P.R.China \\ $\square$ Corresponding authors: Fa-Yu Liu, Department of Oromaxillofacial-Head and Neck, Oral Maxillofacial Surgery, School of Stomatology, China Medical \\ University, No.117, Nanjing Street, Heping District,Shenyang, Liaoning 110002, P.R. China. Telephone: +86-24-22894773; E-mail address: 1fyhjk@126.com or \\ Chang-Fu Sun, Department of Oromaxillofacial-Head and Neck, Oral Maxillofacial Surgery, School of Stomatology, China Medical University, No.117, Nanjing \\ Street, Heping District,Shenyang, Liaoning 110002, P.R. China. Telephone: +86-24-22894773; E-mail address: changfusun@hotmail.com
}

(c) Ivyspring International Publisher. This is an open access article distributed under the terms of the Creative Commons Attribution (CC BY-NC) license (https://creativecommons.org/licenses/by-nc/4.0/). See http://ivyspring.com/terms for full terms and conditions.

Received: 2018.08.28; Accepted: 2019.03.04; Published: 2019.04.25

\begin{abstract}
This study aimed at determining the role of hsa-let-7e-5p in the progression of head and neck squamous cell carcinoma (HNSCC). The relative levels of hsa-let-7e-5p transcripts in 15 paired of HNSCC and adjacent non-tumor tissues and cells were examined by quantitative real-time PCR (qRT-PCR). The potential targets of hsa-let-7e-5p were predicted and validated by luciferase assay. The impact of altered hsa-let-7e-5p expression on HNSCC cell proliferation and metastasis was determined by CCK-8, wound healing, transwell migration and invasion assays. The effect of hsa-let-7e-5p over-expression on the growth of HNSCC was examined in vivo. Hsa-let-7e-5p expression was significantly down-regulated in HNSCC tissues and highly metastatic PCl-37B cells. Bioinformatic analysis predicted that hsa-let-7e-5p bound to the 3'untranslated region (3'UTR) of chemokine receptor 7(CCR7), which was validated by luciferase assay. While transfection with hsa-let-7e-5p mimic significantly decreased CCR7 protein expression, transfection with hsa-let-7e-5p inhibitor increased CCR7 protein expression in HNSCC cells. Similarly, hsa-let-7e-5p over-expression inhibited PCl-37B cell proliferation, wound healing, migration and invasion, while inhibition of endogenous hsa-let-7e-5p had opposite effects in PCl-37A cells. Hsa-let-7e-5p over-expression inhibited PCl-37B tumor growth in vivo. Therefore, hsa-let-7e-5p acts as a tumor suppressor to inhibit the progression of HNSCC by targeting CCR7 expression. Hsa-let-7e-5p and CCR7 may be therapeutic targets of HNSCC.
\end{abstract}

Key words: head and neck squamous cell carcinoma (HNSCC), chemokine receptor 7(CCR7), hsa-let-7e-5p, proliferation, metastasis

\section{Introduction}

Head and neck squamous cell carcinoma (HNSCC) is a commonly malignant tumor in the region [1]. Despite intensive researches and improvements in therapies, including surgery, radiation and chemotherapy, the five-year survival rate of HNSCC patients remains low due to its high local and distant metastasis [2]. Therefore, understanding the molecular mechanisms underlying the metastasis of HNSCC will be important for identifying the potential targets to develop therapies for HNSCC patients.

MicroRNAs can bind to the 3'untranslational region ( $\left.3^{\prime} \mathrm{UTR}\right)$ of their target mRNAs to promote their degradation and inhibit their translation [3,4]. Currently, the role of hsa-let-7e-5p remains controversial. While down-regulated hsa-let-7e-5p expression is related to aggressiveness and poor prognosis of breast cancer [5], non-small-cell lung cancer (NSCLC) [6] and epithelial ovarian cancer [7], 
other studies reported that hsa-let-7e-5p expression is up-regulated in hepatocellular carcinoma [8], colon cancer [9] and esophageal cancer [10]. Recent studies have revealed that hsa-let-7e-5p expression is up-regulated in oral tongue squamous cell carcinoma, particularly in some young cancer patients [11] and up-regulated hsa-let-7e-5p expression promotes the invasion and metastasis of esophageal squamous cell carcinoma [10]. Hence, what is the hsa-let-7e-5p expression pattern in HNSCC and how hsa-let-7e-5p regulates the progression and metastasis of HNSCC are unclear.

In this study, the levels of hsa-let-7e-5p expression in paired HNSCC and adjacent non-tumor tissues and HNSCC cells were examined. Furthermore, the effect of altered hsa-let-7e-5p expression on HNSCC cell proliferation, wound healing, migration and invasion in vitro and the impact of hsa-let-7e-5p over-expression on the HNSCC growth in vivo were investigated. The results indicated that hsa-let-7e-5p expression was down-regulated in HNSCC tissues and cells and hsa-let-7e-5p acted as a tumor suppressor to inhibit the progression and metastasis of HNSCC by targeting CCR7 expression.

\section{Materials and Methods}

\section{Cell culture and transfection}

HNSCC PCI-37B and PCI-37A cells were derived from a patent with lymph node metastatic and primary HNSCC, respectively and were gifts from the University of Pittsburgh Cancer Institute. PCI-37B cells express higher levels of CCR7 mRNA transcripts and have a high metastatic ability while PCI-37A cells express lower levels of CCR7 mRNA transcripts with a low metastatic ability [12]. The cells were cultured in DMEM medium (Invitrogen, Carlsbad, USA) supplemented with $10 \%$ fetal bovine serum (FBS, Gibco, Carlsbad, USA), $100 \mathrm{U} / \mathrm{ml}$ of penicillin and $100 \mu \mathrm{g} / \mathrm{ml}$ of streptomycin at $37^{\circ} \mathrm{C}$ in a $5 \% \mathrm{CO}_{2}$ incubator.

PCI-37B cells $\left(3 \times 10^{5}\right.$ cells/well) were cultured in 6-well plates overnight and transfected with control scramble or hsa-let-7e-5p mimic (Genepharma, Suzhou and Wanleibio, Shenyang, China, respectively) using Lipofectamine 2000 (Invitrogen). Similarly, PCI-37A cells were transfected with control scramble or hsa-let-7e-5p-specific inhibitor (Genepharma). Two days after transfection, the efficacy of hsa-let-7e-5p over-expression and silencing was examined by qRT-PCR.

\section{Clinical human tumor tissues}

Surgical HNSCC tumor and matched adjacent non-tumor tissues were obtained from 15 patients, who underwent surgical resection prior to chemotherapy or radiotherapy at the Department of
Oral and Maxillofacial Surgery, School and Hospital of Stomatology, China Medical University (Table 1). Written informed consent was obtained from individual patients before surgery and the experimental protocol was approved by the Ethical Committee of China Medical University. The tissues were immediately stored in liquid nitrogen before process.

\section{Total RNA extraction and qRT-PCR}

Total RNA was extracted from HNSCC cells and tissues using the high purity total RNAs rapid extraction kit (BioTeke, Beijing, China) following the manufacturer's protocol. After being qualified and quantified, the RNA samples were reversely transcribed into cDNAs using the High Capacity cDNA Reverse Transcription Kit (Thermofisher). The relative levels of hsa-let-7e-5p to control U6 transcripts in each sample were tested by qRT-PCR using the SYBR Green mixture (Solarbio, Beijing, China) and specific primers in Superior 5-color Quantitative Real-Time PCR system (Bioneer, KOR). The primer sequences are shown in Table 2. The data were analyzed by the $2^{-\Delta \Delta C t}$ method.

Table 1. Clinical and pathological features of 15 HNSCC patients.

\begin{tabular}{llllll}
\hline $\begin{array}{l}\text { Patient } \\
\text { number }\end{array}$ & $\begin{array}{l}\text { age(year)/ Tumor site } \\
\text { sex }\end{array}$ & $\begin{array}{l}\text { Differentiation } \\
\text { degree of the } \\
\text { tumor }\end{array}$ & $\begin{array}{l}\text { Metastatic } \\
\text { lymph node }\end{array}$ & TNM \\
\hline 1 & $59 / \mathrm{M}$ & Buccal & Medium & Negative & T2N0M0 \\
2 & $54 / \mathrm{M}$ & $\begin{array}{l}\text { Tongue and } \\
\text { Soft Palate }\end{array}$ & High & Negative & T2N0M0 \\
3 & $64 / \mathrm{F}$ & Buccal & Medium & Negative & T2N0M0 \\
4 & $50 / \mathrm{M}$ & Tongue & Medium & Positive & T2N1M0 \\
5 & $66 / \mathrm{F}$ & Buccal & Medium & Positive & T4N1M0 \\
6 & $54 / \mathrm{F}$ & Tongue & Medium & Negative & T2N0M0 \\
7 & $51 / \mathrm{M}$ & Mouth Floor & Poorly & Negative & T2N0M0 \\
8 & $47 / \mathrm{M}$ & Soft Palate & Medium & Negative & T1N0M0 \\
9 & $68 / \mathrm{M}$ & Tongue & Medium & Negative & T2N0M0 \\
10 & $61 / \mathrm{M}$ & Gingival & Medium & Positive & T1N2bM0 \\
11 & $72 / \mathrm{M}$ & Tongue & Poorly & Negative & T2N0M0 \\
12 & $63 / \mathrm{M}$ & Tongue & Poorly & Positive & T2N2cM0 \\
13 & $61 / \mathrm{F}$ & Tongue & Poorly & Negative & T2N0M0 \\
14 & $58 / \mathrm{M}$ & Tongue & Medium & Negative & T2N0M0 \\
15 & $50 / \mathrm{M}$ & Tongue & Medium & Negative & T2N0M0 \\
\hline
\end{tabular}

Table 2. Oligonucleotide primer sets for real-time PCR

\begin{tabular}{|c|c|c|c|c|}
\hline name & Sequence $\left(5^{\prime} \neg 3^{\prime}\right)$ & Length & $\mathrm{Tm}$ & size \\
\hline hsa-let-7e-5p F & $\begin{array}{l}\text { AGCAAGCTTTGGCACCCACC } \\
\text { CGTAGAAC }\end{array}$ & 28 & 55.0 & 400 \\
\hline hsa-let-7e-5p R & $\begin{array}{l}\text { TAAGGATCCGATGCAGGGAC } \\
\text { AAGGACAGAA }\end{array}$ & 30 & 55.0 & \\
\hline U6 F & CTCGCTTCGGCAGCACA & 17 & 60.4 & 94 \\
\hline U6 R & AACGCTTCACGAATTTGCGT & 20 & 59.7 & \\
\hline CCR7-3'UTR-WT F & $\begin{array}{l}\text { CTAGCTAGCTGGACTAGAGG } \\
\text { GACCTC }\end{array}$ & 26 & 55 & 374 \\
\hline CCR7-3'UTR-WT R & $\begin{array}{l}\text { ACGCGTCGACTCATTCAGAG } \\
\text { GACTC }\end{array}$ & 25 & & \\
\hline CCR7-3'UTR-MT F & AGCTTGGTCAACCAATGCCA & 20 & 58 & 374 \\
\hline CCR7-3'UTR-MT R & TTGACCAAGCTGGGATTGGG & 20 & & \\
\hline
\end{tabular}




\section{Bioinformatics analysis}

The potential targets of hsa-let-7e-5p were predicted by bioinformatic analysis using online websites (targetScanSites, picTarSites, miRandaSites).

\section{Dual-luciferase reporter assay}

The wild type (WT) and mutant type (MT) of CCR7 3'UTR sequences were generated by PCR amplification from human genomic DNA using specific primers (Table 2) and cloned into pmirGLO- reporter (Promega), respectively. PCI-37B cells were transfected with either plasmid for the WT or MT of CCR7 3' UTR luciferase reporter, together with control scramble or hsa-let-7e-5p mimic as well as the plasmid for Renilla luciferase. Two days later, the luciferase activities were measured using Dual Luciferase Reporter Assay System (Promega). The firefly luciferase activity was normalized to the renilla luciferase activity.

\section{Western blot}

The cells in different groups were harvested and lyzed in RIPA lysis buffer, followed by centrifuging at $4^{\circ} \mathrm{C} 12,000 \mathrm{~g}$ for $10 \mathrm{~min}$. The concentrations of total proteins in individual lysate samples were measured using the BCA protein concentration assay kit (Wanleibio, Shenyang, China). Individual lysates (40 $\mu \mathrm{g}$ /lane) were separated by sodium dodecyl sulfate polyacrylamide-gel-electrophoresis on $10 \%$ gels and transferred onto polyvinylidene difluoride membrane (Millipore, USA). The membranes were treated with $5 \%$ fat-free dry milk in TBST and incubated with rabbit anti-CCR7 (Bioyanbio, Zhenjing, China) and anti- $\beta$-actin antibodies (Wanleibio, Shenyang, China) at $4^{\circ} \mathrm{C}$ overnight. The membranes were treated with horseradish peroxidase-conjugated goat anti-rabbit IgG (Wanleibio, Shenyang, China) and visualized by the enhanced chemiluminescent reagent, followed by imaging in the Gel Imaging System (LiuYi, Beijing, China). The relative levels of CCR7 to $\beta$-actin protein expression were analyzed using Gel-Pro-Analyzer software 6.0 (Media Cybernetics US).

\section{Cell proliferation assay}

The different groups of HNSCC cells $\left(3 \times 10^{3}\right.$ cells/well) were cultured in triplicate in 96-well plates for 4 days. The cell proliferation in individual groups was examined longitudinally using the CCK8 proliferation assay kit. Briefly, during the last 2-h culture, the cells were treated with the CCK8 reagent and the absorbance of individual wells of cells were measured at $450 \mathrm{~nm}$ using a microplate spectrometer readers (Biotek, US).

\section{Wound-healing assay}

The cells in different groups were cultured in triplicate in 6-well plates up to $90 \%$ confluency, and the monolayer of cells was wounded using a micropipette tip. We used Mitomycin $C$ and medium without FBS to culture cells to discard the possible influence of proliferation. After being washed, the cells were cultured for $0 \mathrm{~h}, 12 \mathrm{~h}$ and $24 \mathrm{~h}$. The wound distance was photographed and the percent migration of individual groups of cells was calculated.

\section{Cell migration and invasion assay}

The different groups of cells $\left(1 \times 10^{4}\right.$ cells/well or $4 \times 10^{3}$ cells/well) were cultured in triplicate in $200 \mu \mathrm{l}$ of serum-free medium in the upper chambers of 24-well transwell chambers ( $8 \mu \mathrm{m}$ pore, Corning, US). The lower chambers were added with $800 \mu$ l of DMEM containing 20\% FBS. One day later, the non-migrated HNSCC cells on the surface membrane of the upper chambers were removed using a cotton swab and the migrated cells on the bottom surface of the upper chambers were fixed and stained with $0.05 \%$ crystal violet, followed by photographed under an inverted microscope (200×) (AE31, Motic, China). The migrated cells in in five random fields were counted in blinded manner.

Similar procedures were used for testing invasion, except that the upper chambers were coated with matrigel and $2 \times 10^{4}$ cells/well or $8 \times 10^{3}$ cells/well were used.

\section{Lentivirus transduction}

PCI-37B cells were cultured in 6-well plates up to $70 \%$ of confluency and transduced with lentivirus for LV-hsa-let-7e-5p mimic or control miRNA Genepharma for $24 \mathrm{~h}$. The cells were used for subsequent experiments.

\section{Xenograft tumor model}

Female BALB/c nude mice (6-week-old) were from Wanleibio and housed in a specific pathogenfree facility with free access to autoclaved foods and water. The mice were randomized and inoculated subcutaneously with $1 \times 10^{7}$ PCI-37B/hsa-let-7e-5p or control PCI-37B/con cells into their right flank. The tumor growth in individual mice was monitored every three days up to 21 days post inoculation. The tumor volumes were calculated by the formula, volume $=$ length $\times$ width $^{2} \times 0.5$. At the end of the experiment, the mice were sacrificed and their tumors were dissected and measured. One piece of tumor sample was used for total RNA extraction to quantify the expression of hsa-let-7e-5p using qRT-PCR.

\section{Statistical analysis}

Data are expressed as mean \pm standard deviations (SD). The difference among the groups was statistically analyzed by one-way analysis of variance 
and the difference between the groups was analyzed by Student's $\mathrm{T}$ test using SPSS 13.0 software. A p-value of $<0.05$ was considered statistically significant.

\section{Results}

\section{Hsa-let-7e-5p is deregulated and targets CCR7 in $\mathrm{HNSCC}$}

To study the role of hsa-let-7e-5p in the development and progression of HNSCC, the relative levels of hsa-let-7e-5p transcripts in 15 paired of HNSCC and adjacent non-tumor tissues were examined by qRT-PCR. The relative levels of hsa-let-7e-5p transcripts in 10 out of 15 HNSCC tissues were significantly lower than that in the adjacent non-tumor tissues, indicated that hsa-let-7e-5p expression was down-regulated in HNSCC $(\mathrm{p}<0.01$, Fig. 1A). Similarly, the relative levels of hsa-let-7e-5p transcripts in the highly metastatic PCI-37B cells were also significantly lower than that in the lowly metastatic PCI-37A cells ( $\mathrm{p}<0.01$, Fig. 1B).

B

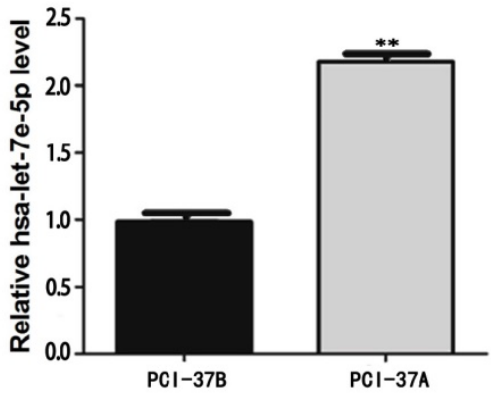

C

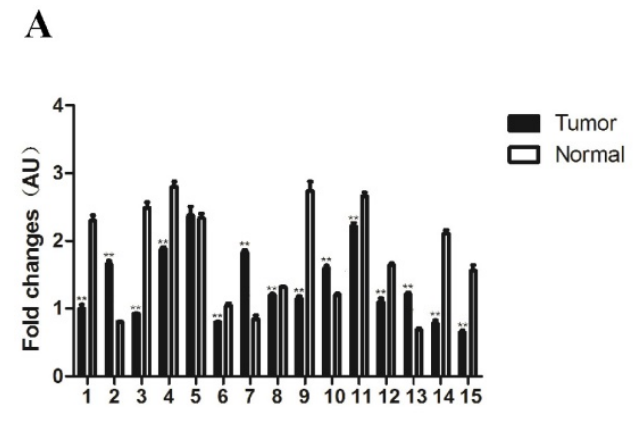

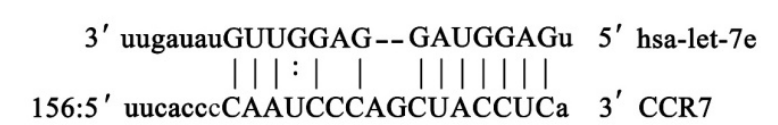

mirSVR score: $\quad-0.4633$

phastCons score: 0.5154

D

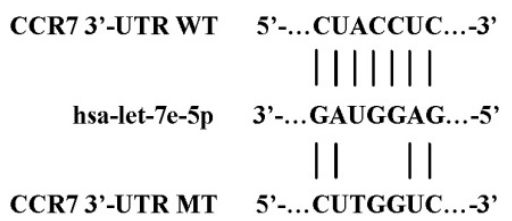

$\mathbf{F}$
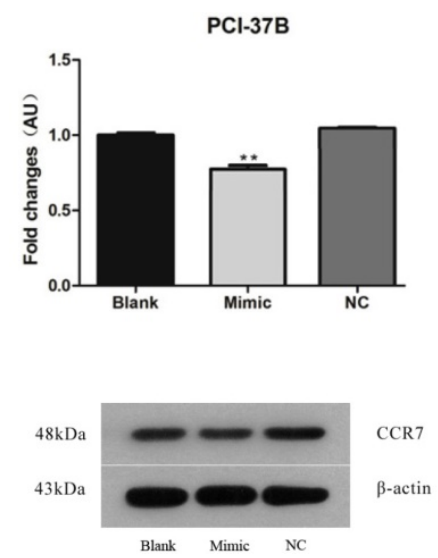

E

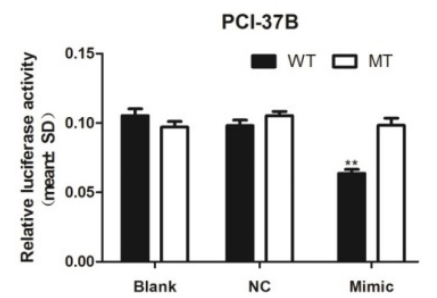

G
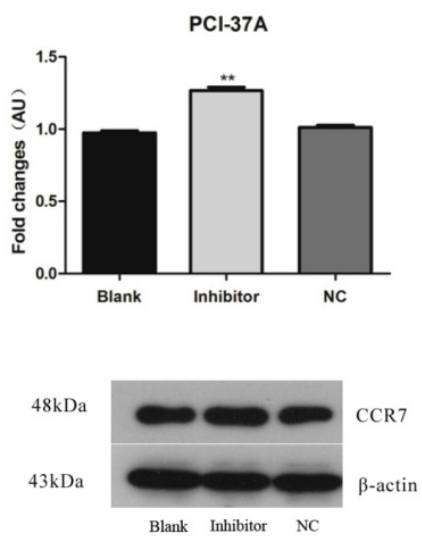

Figure 1. Hsa-let-7e-5p expression is down-regulated in HNSCC tissues and cells and targets CCR7. The relative levels of hsa-let-7e-5p expression in 15 paired of HNSCC and adjacent non-tumor tissues (A) and cells (B) were determined by QRT-PCR. (C) The potential sequence in the 3'UTR CCR7 for hsa-let-7e-5p binding was predicated by bioinformatics. (D) The sequences of the wild-type (WT) and mutant (MT) 3'UTR CCR7 for hsa-let-7e-5p binding. (E) Dual-luciferase reporter assay revealed that transfection with hsa-let-7e-5p mimic significantly reduced the WT, but not the MT, 3'UTR CCR7-regulated luciferase expression in PCl-37B cells. (F) Transfection with hsa-let-7e-5p mimic significantly decreased CCR7 protein expression in PCl-37B cells. (G) Transfection with hsa-let-7e-5p inhibitor significantly increased CCR7 protein expression in PCI-37A cells. Data are representative images or expressed as the mean \pm SD of each group from three separate experiments. **p $<0.01$. 
To explore how hsa-let-7e-5p acts in HNSCC, the potential targets of hsa-let-7e-5p were predicted by bioinformatics analysis. The 3'UTR of CCR7 contained the sequence for hsa-let-7e-5p binding (Fig. 1C). Next, the mutant 3'UTR of CCR7 was synthesized (Fig. 1D) and the potential binding of hsa-let-7e-5p to the 3'UTR of CCR7 and its function were validated by dual-luciferase reporter assays. As shown in Figure 1E, co-transfection with hsa-let-7e-5p mimic and the plasmid for wild-type of CCR7 3'UTR-regualted luciferase significantly reduced the luciferase activities in PCI-37B cells, relative to the controls transfected with hsa-let-7e-5p mimic and the plasmid for the mutant CCR7 3'UTR-regulated luciferase and the cells transfected with the plasmid and control miRNA (NC) or the cells transfected with the plasmid alone $(p<0.01)$. Western blot analysis indicated that transfection with hsa-let-7e-5p mimic significantly decreased the levels of CCR7 protein expression in PCI-37B, whereas transfection with hsa-let-7e-5p inhibitor significantly increased the CCR7 protein expression in PCI-37A (Fig. 1F and 1G). Collectively, such data indicate that down-regulated hsa-let-7e-5p expression is associated with the progression of HNSCC and suggest that hsa-let-7e-5p may bind to the 3'UTR of CCR7 to decrease its protein expression in HNSCC cells.

\section{Altered hsa-let-7e-5p expression modulates the tumor-behaviors in HNSCC cells}

To explore the regulatory function, HNSCC PCI-37B and PCI-37A cells were transfected with control scramble miRNA, hsa-let-7e-5p mimic or inhibitor, respectively. The proliferation, wound healing, migration and invasion of the different groups of cells were examined. In comparison with the unmanipulated (Blank) cells, transfection with control scramble miRNA did not alter the proliferation, wound healing, migration and invasion of both PCI-37B and PCI-37A cells (Fig. 2). Transfection with hsa-let-7e-5p mimic significantly reduced the proliferation, wound healing, migration and invasion of PCI-37B cells ( $\mathrm{p}<0.01$ for all, Fig. 2A, $2 \mathrm{C}, 2 \mathrm{E}$, and 2G). In contrast, transfection with hsa-let-7e-5p inhibitor significantly enhanced the proliferation, wound healing, migration and invasion of PCI-37A cells $(p<0.01$ for all, Fig. 2B, 2D, 2F and $2 \mathrm{H})$. Hence, altered hsa-let-7e-5p expression changed the tumor behaviors of HNSCC cells in vitro.

\section{Hsa-let-7e-5p over-expression inhibits xenograft tumor growth in vivo}

Finally, the impact of hsa-let-7e-5p overexpression on tumor growth was determined. Individual $\mathrm{BALB} / \mathrm{c}$ nude mice were inoculated sub- cutaneously with the unmanipulated, the scramble miRNA-transfected or hsa-let-7e-5p-transfected PCI$37 \mathrm{~B}$ cells and the tumor growth in individual groups of mice was monitored (Fig. 3A and 3B). Induction of hsa-let-7e-5p over-expression significantly inhibited the growth of inoculated HNSCC at 15 days post inoculation and later $(p<0.05$ or $p<0.01)$. Further qRT-PCR revealed that the relative levels of hsa-let$7 e-5 p$ transcripts in the hsa-let-7e-5p over-expressing tumors were significantly higher than that of the controls ( $\mathrm{p}<0.01$, Fig. 3C). Therefore, increased hsalet-7e-5p expression significantly inhibited the growth of inoculated HNSCC in vivo.

\section{Discussion}

Previous studies have shown the levels of has-let-7e-5p expression in different types of malignant tumors remain controversial [6-10]. There is little information on the expression pattern and potential role of has-let-7e-5p in HNSCC. In this study, we found that has-let-7e-5p transcription was down-regulated in HNSCC tissues and highly metastatic HNSCC cells. These data suggest that has-let-7e-5p may act as a tumor suppressor and its down-regulated expression may promote the development and progression of HNSCC, extending previous observations in NSCLC [13], breast cancer [5] and glioma [14]. However, our data were in disagreement with other reports that up-regulated has-let-7e-5p expression is associated with aggressiveness of oral tongue and esophageal squamous cell carcinoma, particularly for young patients [11]. Actually, we found that has-let-7e-5p expression was significantly up-regulated in 4 out of 15 HNSCC. The difference may stem from different population of patients between our study and those of others. These contradictory findings suggest that has-let-7e-5p expression may be regulated by specific tumor type, stage and its host age and has-let-7e-5p may have different roles during the pathogenesis of different types of malignant tumors by targeting different gene expression.

MicroRNAs function as tumor suppressors or oncogenes to inhibit or promote the development and progression of malignant tumors. While hsa-let-7e-5p can inhibit the proliferation and metastasis of glioma stem cells [14] and colorectal cancer [15], hsa-let$7 e-5 p$, together with miR-99b and miR-125a, acts as a pro-metastasis oncomir to promote ESCC cell migration and invasion in vitro [10]. We found that induction of hsa-let-7e-5p over-expression inhibited the proliferation, wound healing, migration and invasion of highly aggressive PCI-37B cells while inhibition of endogenous hsa-let-7e-5p expression by transfection with hsa-let-7e-5p inhibitor enhanced the 
proliferation, wound healing, migration and invasion of less aggressive PCI-37A cells. Furthermore, hsa-let-7e-5p over-expression retarded the growth of inoculated PCI-37B HNSCC in mice. There independent lines of evidence demonstrated that hsa-let-7e-5p acted as a tumor suppressor to inhibit the development and progression of HNSCC. We are interested in further investigating the impact of altered hsa-let-7e-5p expression in the lymph node and distant metastasis of HNSCC in vivo in the future. If validated, functional increase in the levels of hsa-let-7e-5p expression may be valuable to inhibit the progression of HNSCC.

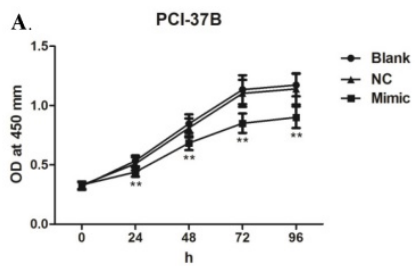

B.
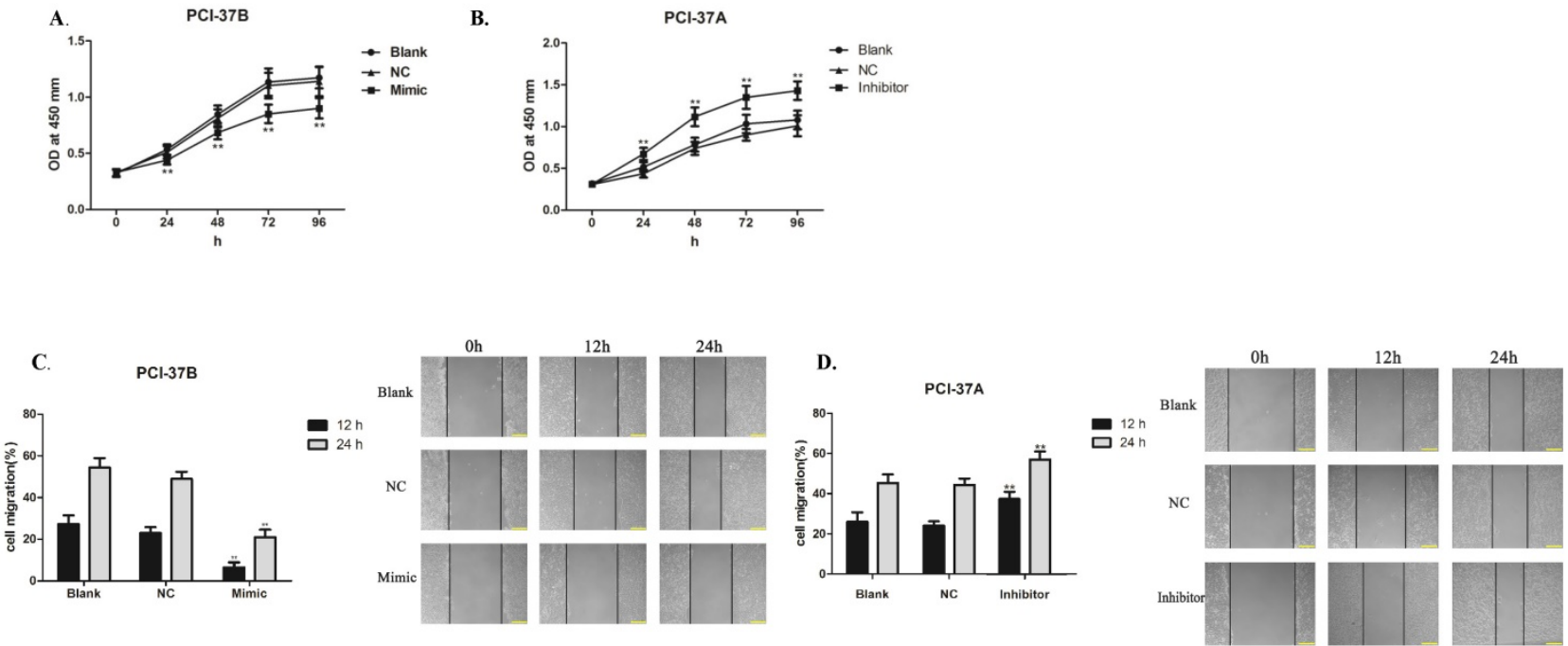

E.
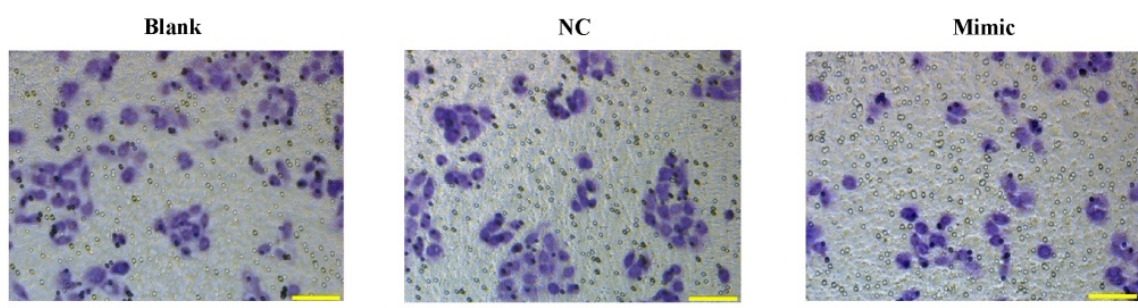

F.
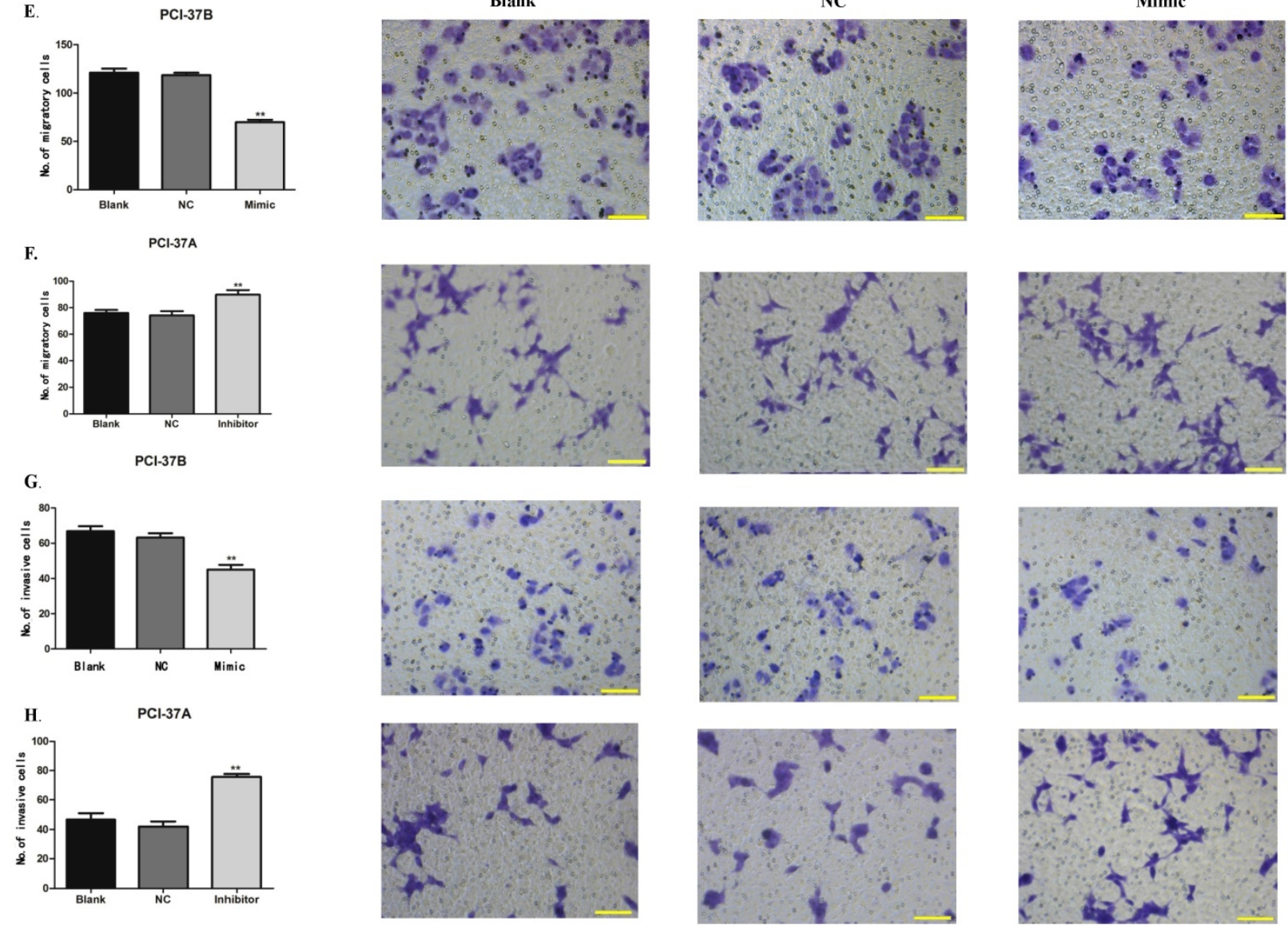

Figure 2. Altered hsa-let-7e-5p expression modulates the behaviors of HNSCC cells in vitro. PCl-37B and PCl-37A cells were unmanipulated or transfected with control scramble, hsa-let-7e-5p mimic or inhibitor, respectively. The proliferation (A, B), wound healing (C, D), migration (E, $F)$ and invasion (G, $H$ ) of each group of cells were examined, respectively. Data are representative images or expressed as the mean \pm SD of each group from three separate experiments (magnification x 100 for wound healing; $\times 200$ for migration and invasion). Bar $=200 \mu \mathrm{m}$. $* * \mathrm{P}<0.01$. 
A

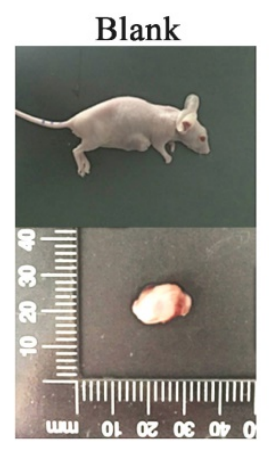

B
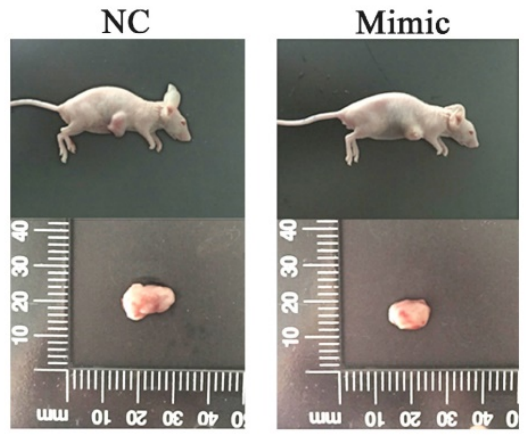

$\mathrm{C}$

PCI-37B
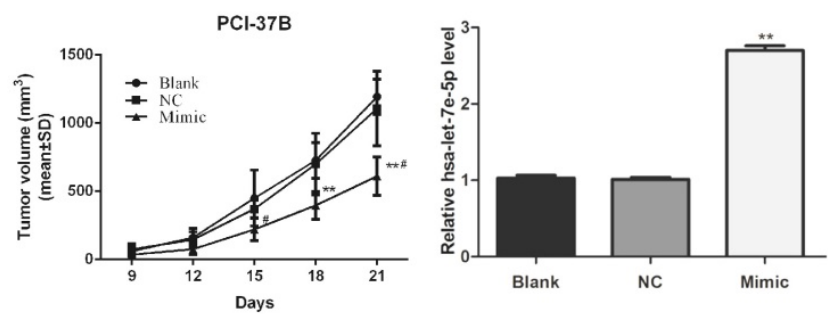

Figure 3. Induction of hsa-let-7e-5p over-expression inhibits the growth of inoculated PCI-37B HNSCC in mice. Individual BALB/c nude mice were injected subcutaneously with unmanipulated, lentivirus-mediated control miRNA-expressing, or has-let-7e-5p over-expressing $\mathrm{PCl}-37 \mathrm{~B}$ cells $\left(1 \times 10^{7}\right.$ cells/mouse). The dynamic growth of inoculated tumors in each group was monitored up to 21 days post inoculation $(A)$. Subsequently, the tumors were dissected out and imaged (B). * $\mathrm{P}<0.05$, ** $\mathrm{P}<0.01$ vs. the Blank. \#P $<$ 0.05 vs. the NC. The relative levels of has-let-7e-5p transcripts in each group of tumors were examined by qRT-PCR (C). Hsa-let-7e-5p expression was assessed by real-time PCR. ** $P<0.01$ vs. the Blank or NC. Data are representative images or present as the mean \pm SD of each group ( $n=6$ per group) from three separate experiments.

To understand the molecular mechanisms underlying the action of hsa-let-7e-5p in inhibiting the progression of HNSCC, we performed bioinformatics to predict the potential targets of hsa-let-7e-5p. We found that the 3'UTR HMGA2, STAT3, and CCR7 contained the potential binding sequence of hsa-let-7e-5p. Further lucirase reporter assay indicated that transfection with hsa-let-7e-5p mimic significantly reduced the WT 3'UTR, but not the MT $3^{\prime}$ UTR of CCR7-regulated luciferase expression in PCI-37B cells. Functionally, hsa-let-7e-5p overexpression decreased the levels of CCR7 protein expression in PCI-37B cells while inhibition of endogenous hsa-let-7e-5p expression increased the levels of CCR7 protein expression in PCI-37A cells. Together, such findings indicated that hsa-let-7e-5p inhibited CCR7 expression by binding to the 3'UTR CCR7 and the CCR7 was one of its targets in HNSCC cells. CCR7 is a member of the G-protein-coupled receptors (GPCR) family, and substantially expressed on several types of immune cells. CCR7 is crucial for chemoattaction of immune cells and tumor cells. Our data were consistent with a recent report and support the notion that high levels of CCR7 expression promote the invasion and metastasis of HNSCC [16] and esophageal squamous cell carcinoma [17]. In addition, our previous studies and those of others have shown that CCR7 can regulate the metastasis of HNSCC through the signaling pathways, including the CCR7-PI3K-Cdc42-Rac [18], CCR7-PKC ${ }_{\alpha}-\mathrm{NF}-\mathrm{KB}$ [19], CCR7-PI3K-Akt-mTOR-NF-kB [20, 21], CCR7JAK2-STAT3 [22], CCR7-MAPKs [23], CCR7-RhoA/ ROCK-Pyk2 cofilin [24-26]. Moreover, the CCR7related signaling can up-regulate some stimulators to enhance the proliferation and angiogenesis of malignant tumors [27]. It is possible that the down-regulated hsa-let-7e-5p expression to enhance CCR7 expression may promote the progression and metastasis of HNSCC thorought similar signal pathways. Thus, such findings may shed new light on regulating CCR7 expression and HNSCC metastasis. We recognized that our study had limitations, including small sample size, the lack of further functional studies on whether over-expression of CCR7 can antagonist the hsa-let-7e-5p mediated inhibition to restore the progression of HNSCC. Therefore further studies in a bigger population are warrented to validate the role of hsa-let-7e-5p in the development and progression of HNSCC.

In conclusion, the results revealed that hsa-let$7 e-5 p$ expression was down-regulated in HNSCC tissues and highly aggressive cells. The hsa-let-7e-5p bound to the 3'UTR CCR7 and inhibited its expression. Hsa-let-7e-5p inhibited the proliferation, wound healing, migration and invasion of HNSCC cells in vitro and the growth of HNSCC tumors in vivo. Collectively, such novel data indicated that hsa-let-7e-5p inhibited the progression and metastasis of HNSCC by targeting CCR7. Such findings may provide new insights into regulating CCR7 expression and HNSCC metastasis.

\section{Abbreviations}

3'UTR: 3'untranslated region; CCR7: Chemokine receptor 7; Blank: Blank control; NC: Negative control; qRT-PCR: quantitative real-time PCR; WT: the wild type; MT: mutant type.

\section{Acknowledgements}

This study was supported by the grants from the National Natural Science Foundation of China (No.81372877), the Basic Scientific Research Projects of Colleges of Liaoning Province (No.LQNK201725), the Key Research and Development Guidance Program in Liaoning Province (No.2017225037), the Scientific Project of Shenyang City (No.17-230-9-12), the Youth Science and Technology Innovation Plan of Shenyang City (No.RC170489). 


\section{Competing Interests}

The authors have declared that no competing interest exists.

\section{References}

1. Yoon Y, Liang Z, Zhang X, Choe M, Zhu A, Cho HT, et al. CXC chemokine receptor-4 antagonist blocks both growth of primary tumor and metastasis of head and neck cancer in xenograft mouse models. Cancer research. 2007; 67: 7518-24.

2. Pulte D, Brenner H. Changes in survival in head and neck cancers in the late 20th and early 21st century: a period analysis. The oncologist. 2010; 15: 994-1001.

3. Bartel DP. MicroRNAs: genomics, biogenesis, mechanism, and function. Cell. 2004; 116: 281-97.

4. Huang X, Liang M, Dittmar R, Wang L. Extracellular microRNAs in urologic malignancies: chances and challenges. International journal of molecular sciences. 2013; 14: 14785-99.

5. Mitra D, Das PM, Huynh FC, Jones FE. Jumonji/ARID1 B (JARID1B) protein promotes breast tumor cell cycle progression through epigenetic repression of microRNA let-7e. The Journal of biological chemistry. 2011; 286: 40531-5.

6. Zhu WY, Luo B, An JY, He JY, Chen DD, Xu LY, et al. Differential expression of miR-125a-5p and let-7e predicts the progression and prognosis of non-small cell lung cancer. Cancer investigation. 2014; 32: 394-401.

7. Xiao M, Cai J, Cai L, Jia J, Xie L, Zhu Y, et al. Let-7e sensitizes epithelial ovarian cancer to cisplatin through repressing DNA double strand break repair. Journal of ovarian research. 2017; 10: 24

8. Shi W, Zhang Z, Yang B, Guo H, Jing L, Liu T, et al. Overexpression of microRNA let-7 correlates with disease progression and poor prognosis in hepatocellular carcinoma. Medicine. 2017; 96: e7764.

9. Chen W, Lin G, Yao Y, Chen J, Shui H, Yang Q, et al. MicroRNA hsa-let-7e-5p as a potential prognosis marker for rectal carcinoma with liver metastases. Oncology letters. 2018; 15: 6913-24.

10. Ma J, Zhan Y, Xu Z, Li Y, Luo A, Ding F, et al. ZEB1 induced miR-99b/let-7e/miR-125a cluster promotes invasion and metastasis in esophageal squamous cell carcinoma. Cancer letters. 2017; 398: 37-45.

11. Hilly O, Pillar N, Stern S, Strenov Y, Bachar G, Shomron N, et al. Distinctive pattern of let-7 family microRNAs in aggressive carcinoma of the oral tongue in young patients. Oncology letters. 2016; 12: 1729-36.

12. Wang J, Xi L, Hunt JL, Gooding W, Whiteside TL, Chen Z, et al. Expression pattern of chemokine receptor 6 (CCR6) and CCR7 in squamous cell carcinoma of the head and neck identifies a novel metastatic phenotype. Cancer research. 2004; 64: 1861-6.

13. Tsai CH, Lin LT, Wang CY, Chiu YW, Chou YT, Chiu SJ, et al. Over-expression of cofilin-1 suppressed growth and invasion of cancer cells is associated with up-regulation of let-7 microRNA. Biochimica et biophysica acta. 2015; 1852: $851-61$.

14. Gong W, Zheng J, Liu X, Ma J, Liu Y, Xue Y. Knockdown of NEAT1 restrained the malignant progression of glioma stem cells by activating microRNA let-7e. Oncotarget. 2016; 7: 62208-23.

15. Shan Y, Liu Y, Zhao L, Liu B, Li Y, Jia L. MicroRNA-33a and let-7e inhibit human colorectal cancer progression by targeting ST8SIA1. The international journal of biochemistry \& cell biology. 2017; 90: 48-58.

16. Muller A, Sonkoly E, Eulert C, Gerber PA, Kubitza R, Schirlau K, et al. Chemokine receptors in head and neck cancer: association with metastatic spread and regulation during chemotherapy. International journal of cancer. 2006; 118: 2147-57.

17. Ding $Y$, Shimada $Y$, Maeda M, Kawabe A, Kaganoi J, Komoto I, et al Association of CC chemokine receptor 7 with lymph node metastasis of esophageal squamous cell carcinoma. Clinical cancer research. 2003; 9: 3406-12.

18. Zhao ZJ, Liu FY, Li P, Ding X, Zong ZH, Sun CF. CCL19-induced chemokine receptor 7 activates the phosphoinositide- 3 kinase-mediated invasive pathway through Cdc42 in metastatic squamous cell carcinoma of the head and neck. Oncology reports. 2011; 25: 729-37.

19. Zhen-jin Z, Peng L, Fa-yu L, Liyan S, Chang-fu S. PKCalpha take part in CCR7/NF-kappaB autocrine signaling loop in CCR7-positive squamous cell carcinoma of head and neck. Molecular and cellular biochemistry. 2011; 357: $181-7$

20. Liu FY, Zhao ZJ, Li P, Ding X, Zong ZH, Sun CF. Mammalian target of rapamycin (mTOR) is involved in the survival of cells mediated by chemokine receptor 7 through PI3K/Akt in metastatic squamous cell carcinoma of the head and neck. The British journal of oral \& maxillofacial surgery. 2010; 48: 291-6.

21. Liu FY, Zhao ZJ, Li P, Ding X, Guo N, Yang LL, et al. NF-kappaB participates in chemokine receptor 7-mediated cell survival in metastatic squamous cell carcinoma of the head and neck. Oncology reports. 2011; 25: 383-91.

22. Liu FY, Safdar J, Li ZN, Fang OG, Zhang X, Xu ZF, et al. CCR7 regulates cell migration and invasion through JAK2/STAT3 in metastatic squamous cell carcinoma of the head and neck. BioMed research international. 2014; 2014: 415375 .
23. Liu FY, Safdar J, Li ZN, Fang QG, Zhang X, Xu ZF, et al. CCR7 regulates cell migration and invasion through MAPKs in metastatic squamous cell carcinoma of head and neck. International journal of oncology. 2014; 45: 2502-10.

24. Yang L, Liu F, Xu Z, Guo N, Zheng X, Sun C. Chemokine receptor 7 via proline-rich tyrosine kinase-2 upregulates the chemotaxis and migration ability of squamous cell carcinoma of the head and neck. Oncology reports. 2012; 28: 1659-64.

25. Xu Z, Zheng X, Yang L, Liu F, Zhang E, Duan W, et al. Chemokine receptor 7 promotes tumor migration and invasiveness via the RhoA/ROCK pathway in metastatic squamous cell carcinoma of the head and neck. Oncology reports. 2015; 33: 849-55.

26. Yue Y, Li ZN, Fang QG, Zhang X, Yang LL, Sun CF, et al. The role of Pyk2 in the CCR7-mediated regulation of metastasis and viability in squamous cell carcinoma of the head and neck cells in vivo and in vitro. Oncology reports. 2015; 34: 3280-7

27. Chi BJ, Du CL, Fu YF, Zhang YN, Wang RW. Silencing of CCR7 inhibits the growth, invasion and migration of prostate cancer cells induced by VEGFC. International journal of clinical and experimental pathology. 2015; 8: 12533-40. 